\title{
A Cloud-Based Energy Management System for Building Managers
}

\author{
Charalampos Marmaras \\ School of Engineering \\ Cardiff University, UK \\ MarmarasC@cardiff.ac.uk
}

\author{
Amir Javed, Omer Rana \\ School of Computer Science \\ and Informatics \\ Cardiff University, UK \\ JavedA7@cardiff.ac.uk
}

\author{
Liana M. Cipcigan \\ School of Engineering \\ Cardiff University \\ CipciganLM@cardiff.ac.uk
}

\begin{abstract}
A Local Energy Management System (LEMS) is described to control Electric Vehicle charging and Energy Storage Units within built environments. To this end, the LEMS predicts the most probable half hours for a triad peak, and forecasts the electricity demand of a building facility at those times. Three operational algorithms were designed, enabling the LEMS to (i) flatten the demand profile of the building facility and reduce its peak, (ii) reduce the demand of the building facility during triad peaks in order to reduce the Transmission Network Use of System (TNUoS) charges, and (iii) enable the participation of the building manager in the grid balancing services market through demand side response. The LEMS was deployed on over a cloud-based system and demonstrated on a real building facility in Manchester, UK.
\end{abstract}

\section{Keywords}

Electric Vehicles, Energy Storage, Energy Management System, Cloud computing, Triad Peak Estimation

\section{INTRODUCTION}

Buildings, in most International Energy Agency (IEA) countries represent $32 \%$ of total energy consumption and $40 \%$ of primary energy consumption [1]. This makes them one of the major contributors to greenhouse gas production $[10,20]$. However, they also provide a great potential for greenhouse gas emission reductions if adequate measures are implemented [18,25]. A significant part of electricity costs in Great Britain (GB) are the TNUo charges, which are calculated by National Grid using the "triad system" [6]. A "triad" refers to the three half-hour periods, separated by atleast ten clear days, when the electricity demand was highest in GB [6]. There is significant interest from the high-consumption customers to know beforehand the "triad" half-hours so they can use energy management techniques to reduce their electricity consumption and their bills [15]. Energy management in buildings is achieved through a building energy management system (BEMS), a computer-based system that controls mechanical and electrical systems to reduce energy cost.

Permission to make digital or hard copies of all or part of this work for personal or classroom use is granted without fee provided that copies are not made or distributed for profit or commercial advantage and that copies bear this notice and the full citation on the first page. Copyrights for components of this work owned by others than ACM must be honored. Abstracting with credit is permitted. To copy otherwise, or republish, to post on servers or to redistribute to lists, requires prior specific permission and/or a fee. Request permissions from permissions@acm.org.

ICPE '17 Companion, April 22-26, 2017, L'Aquila, Italy.

(C) 2017 ACM. ISBN 978-1-4503-4899-7/17/04 ..\$15.00

DOI: http://dx.doi.org/10.1145/3053600.3053613
Considerable literature exists on intelligent BEMSs that utilise sophisticated algorithms to maximise the energy savings while maintaining occupants' comfort. The use of artificial neural networks (ANNs) to control BEMSs has been examined in $[4,5]$, offering self-learning abilities to support both thermal comfort and energy savings in public buildings. Classification techniques were applied to predict the daily electricity consumption for buildings in Birmingham, demonstrating a $99 \%$ accurate prediction [12]. ANNs are also used for the electricity demand forecast of a large office building [14], capturing $97 \%$ of variability in hourly electricity demand (based on weather and electric power consumption alone). Other computational intelligence techniques such as fuzzy c-means clustering, support vector machines, and genetic algorithms have been used for describing the energy consumption behaviour in $[2$, 8].

Recent work has involved finding ways to operate buildings in a "smart" way, considering them as part of local energy systems (microgrid). These local energy systems are aggregating energy storage, Electric Vehicle (EV) charging, PhotoVoltaic (PV) panels and other distributed generators (DGs). In [7], the authors use the general mixed integer programming method to schedule the operation of a PV panel, energy storage and Combined Heat and Power (CHP) unit in order to minimise the total electricity cost of the building. Similar work can be found in [11], while in [3] the authors perform a cost-benefit analysis to calculate the optimal size of battery storage in a microgrid within buildings. The authors of $[13,22]$ have used EVs in order to consume locally the generated power from PVs and reduce the potential impact on the grid. Considering a grid-connected operation of a microgrid within buildings, demand response capabilities have also been incorporated into energy management systems. A review of possible control architectures for a microgrid within buildings can be found in [9]. In [19] the authors present an algorithm that enables the participation in a demand response scheme through utilising home appliances and EVs. For such applications, multi agent systems (MAS) have been developed $[23,26]$. Based on MAS theory, the authors of [24] developed a negotiation agent to facilitate bi-directional energy trading between the microgrid and the grid. A bidding strategy was used in [16], to enable the participation of a microgrid in the day-ahead energy market.

However, existing control models and energy management systems are using only one control strategy, and do not provide flexibility when it comes to other objectives. Consequently, this limits their efficiency and performance in a real application. The building managers require an energy management system which offers flexibility and demonstrates adequate performance regardless of the operational target. Furthermore the "triad" costs are completely neglected despite their significant size (e.g. $£ 45 / \mathrm{kW}$ ), and the 
building managers struggle to reduce their electricity bills. In this paper a complete Local Energy Management System (LEMS) was deployed using a cloud-based infrastructure and demonstrated on a real building facility, to manage the demand from EVs and Energy Storage Units (ESUs) at building premises. The objectives of the LEMS are to (i) flatten the demand profile of the building facility and reduce its peak, (ii) reduce the demand of the building facility during triad peaks in order to reduce the TNUoS charges of the building manager and (iii) enable the participation of the building manager in the grid balancing services market through demand side response.

\section{LEMS}

The proposed LEMS architecture is presented in Fig. 1. The LEMS manages the demand from EVs and ESUs at building premises by sending power set points through a Gateway to EV chargers. A Triad Peak Prediction Tool and an Electricity Demand Forecast Tool were developed to work with the LEMS. The Triad Prediction Tool calculates the probability of having a triad peak in GB and provides warnings for the dates and times that a triad peak is expected to occur. The Electricity Demand Forecast Tool forecasts the electricity demand of the building for that period using an ANN. To generate the triad warnings, the Triad Prediction Tool uses historical triad data available from the System Operator [6]. To forecast the electricity demand of the building, the Electricity Demand Forecast Tool uses historical building demand data and the local weather.

The LEMS operates in timesteps during which the system is considered static (changes are only discovered at the end of the timestep). The timestep duration applied in this work is considered to be 15 minutes. It was concluded that this timestep provides an acceptable trade-off between a dynamic and a reliable operation that allows the frequent capture of the system's condition and minimizes the risk of communication lags. Information regarding all EV's and ESU's is collected at every timestep and stored in a database for future reference.

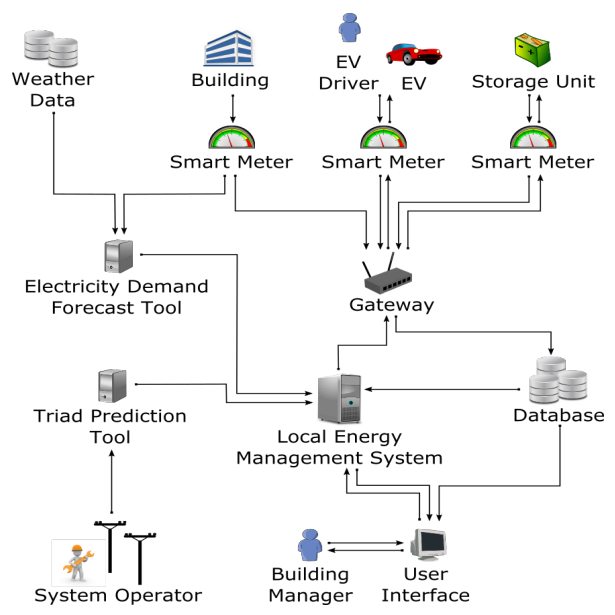

Figure 1: Architecture of LEMS

Triad Peak and Building Energy Prediction: A stochastic model was developed to estimate the "triad" days of the forthcoming year. The model uses historical triad data to calculate the probability $\mathrm{P}(\mathrm{i})$ of having a triad peak on certain dates.

$$
P(i)=\frac{\sum_{n=1}^{N}\left[\sum_{k=1}^{3}\left[n \cdot \frac{1}{\sigma \sqrt{2 \pi}} \cdot e^{-\frac{\left(i-T_{n}^{(k)}\right)^{2}}{2 \sigma^{2}}}\right]\right]}{\sum_{n=1}^{N} 3 n}
$$

where:- $\mathrm{P}(\mathrm{i})$ is the probability of the $i^{\text {th }}$ day including a triad peak, $i$ is the daily index, $n$ is the year index $\left(N^{t h}\right.$ year is the most recent year), $N$ is the total number of years that triad data are available, $k$ is the triad index for a year, $\sigma$ is the standard deviation of the normal distribution ( $=1$ in our model) and $T_{n}^{(k)}$ is the day that includes the kth triad peak of year $n$

As seen from eq. (1), the probability $\mathrm{P}(\mathrm{i})$ of each day $i$ is obtained by superimposing a set of three normal distributions for each year that triad data are available. These normal distributions have as mean value the index of the triad day (i.e. the day on which one of the triad peak occurs) and a standard deviation of 1 . With this method, each triad day affects not only the probability of the same day but also the probability of the neighbouring ones. This is undertaken in order to consider the uncertainties introduced by the weather in the calculations. The model is built with a learning feature which improves the probability assessment on an annual basis when new triad data becomes available. Emphasis is given on the most recent data, by including weights ( $n$ in (1)) that increase as data are available from a more recent year. In this way the model can detect possible changes in the pattern caused by external factors that change on an annual basis (e.g. weather, technology). Finally, the sum of the weighted normal distributions is divided by the sum of weights $n$ in order to obtain the probability $\mathrm{P}(\mathrm{i})$ (weighted average).The model also calculates the most probable half-hours within a day during which the triad peak demand is expected to occur.

Building Electricity Demand Forecast: In this stage data is preprocessed by combining those weather attributes that have high correlation with electricity demand. The preprocessed data is then used to build a forecasting model using the Weka toolkit [17]. The model performs a day-ahead power demand forecast using the model build earlier.

Model Validation: The model was developed in Matlab and trained on real triad data from the period $1990-2014$. The data were obtained from National Grid [6] and included information regarding the dates and times of the triad peaks of the corresponding years. Electricity demand data were available from six commercial buildings in Manchester, UK. Weather data were also available from weather stations in Manchester, UK and were used in Electricity Demand Forecast stages. A triad peak probability warning threshold of $70 \%$ was assumed (the building manager will be warned for periods which constitute the top $30 \%$ of the results). The actual triad peak dates and times of 2014/2015 are presented in Table I [6].

Table 1: The Actual"Triad" Peak Dates and Times of 2014/2015

\begin{tabular}{ll}
\hline \multicolumn{1}{c}{ Triad Date } & Triad HalfHour \\
\hline December 4 2014 & 17:00 -17:30 \\
January 19 2015 & 17:00-17:30 \\
February 2 2015 & 17:30 -18:00 \\
\hline
\end{tabular}

Figure 2 presents the actual triad peak half hours of 2014/2015 compared to the calculated half-hourly probability distribution. As 


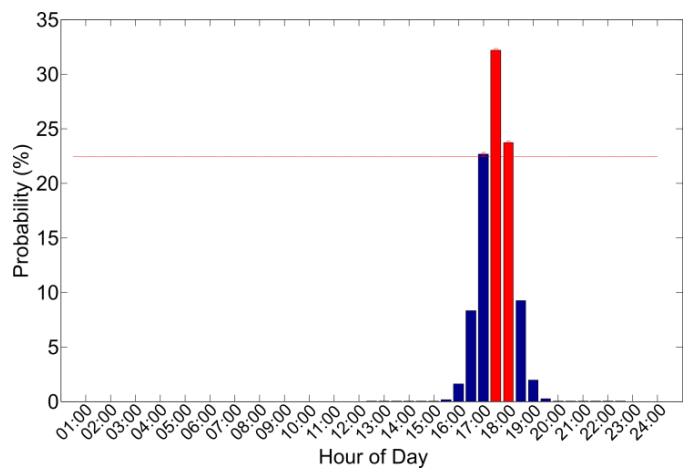

Figure 2: The actual triad peak half-hours of 2014/2015 compared to the calculated half-hourly probability distribution.

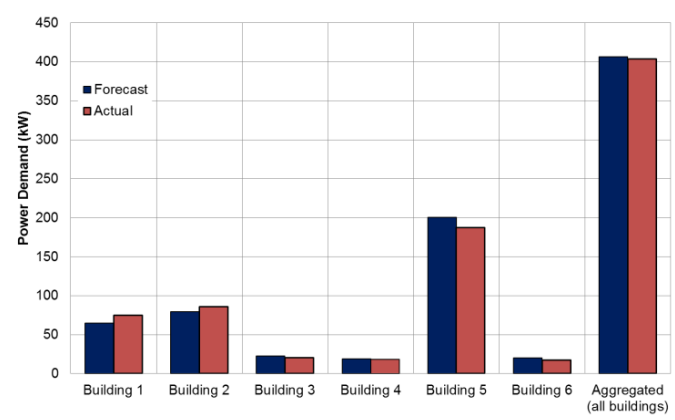

Figure 3: Forecasted and actual power demand for all buildings seen from Table I, two triad peaks occurred between 17:00 and 17:30, while the third one occurred between 17:30 and 18:00. The Triad Probability Assessment model calculated the period between 16:30 and 18:00 to be the most probable for a triad peak which was correct for all three triad peaks. Having an accurate prediction, the building managers are able to avoid the triad peak half-hours and reduce their bills. Furthermore, the fact that the "alert" zone is only 1.5 hours long avoids any other costs associated with the reduction of the building's demand (e.g. the cost of the occupants' discomfort). The power demand at 17:30 of the 6th of December 2013 (actual triad half hour and day) was forecasted separately for each building. The forecasted and actual demand for each building is presented in Fig 3. Looking at the six buildings, the maximum error was found to be $-13.56 \%$ for Building 5 . Three forecasts had errors less than $3 \%$ which are considered very accurate, while the accuracy of the other three were found to be above $89 \%$. Overall, the aggregated demand of all the buildings was forecasted with an error of $-2.422 \%$, which is considered very satisfactory.

\section{ENERGY MANAGEMENT SYSTEM OPE- RATION}

The LEMS maximizes its utility to the building manager by adjusting its operational target (objective) according to the system status and condition. Three scheduling algorithms for the management of the EVs and the ESUs were designed, namely Peak Shaving Schedule, Triad Cost Reduction Schedule and Demand Response Schedule. Each algorithm serves one objective and the LEMS shifts from one scheduling strategy to another depending on the objective set by the building manager.

Peak Shaving Schedule: The Peak Shaving Schedule aims to flatten the aggregated demand profile of the building facility. This is achieved by filling the valleys and shaving the peaks of the demand profile using the controllable loads (EVs, ESUs) of the building facility. The LEMS calculates the charging / discharging schedules of the EVs and ESUs, and sends them the corresponding power set points at the beginning of every timestep. These power set points are messages with the exact power rate at which each EV/ESU must charge/discharge at each timestep. A message of $2.7 \mathrm{~kW}$ sent to EV1, for example, means that in this timestep EV1 must charge at $2.7 \mathrm{~kW}$ from its charger. Similarly, a message of $-1.6 \mathrm{~kW}$ sent to ESU1 means that in this timestep ESU1 must discharge at a rate of $1.6 \mathrm{~kW}$. A ranking function is used in every timestep to identify the most preferable timesteps for charging / discharging. The output of this function are two sets of weights $(\mathrm{r} 1(\mathrm{t})$ and $\mathrm{r} 2(\mathrm{t}))$ indicating the preference order for charging and discharging respectively, according to the aggregated scheduled demand of the building facility. Lower weights are given to the most preferred timesteps (the most preferred timestep has a weight of 1 etc.) and vice versa. To schedule the charging/discharging of the EVs, the LEMS calculates the charging/discharging power set points by solving (2) upon the connection of an EV. It was assumed that the EV drivers agree to provide a certain discharging capacity (V2B) to the LEMS.

$$
\min \sum_{t=t_{0}}^{t_{0}+C P}\left(P_{D}(t) \cdot r_{1}(t)+P_{c}(t) \cdot r_{2}(t)\right)
$$

with the following constraints:

$\sum_{t=t_{0}}^{t_{0}+C P} T \cdot P_{C}(t)=\frac{\left(S o C_{\max }-S o C_{i n}\right) \cdot B C}{C_{e f f}}$

$\sum_{t=t_{0}}^{t_{0}+C P} T \cdot P_{D}(t)=\frac{V 2 B \cdot B C}{C_{\text {eff }}}$

$P_{D}(t) \leq P_{n}$

$P_{C}(t) \leq P_{n}$

$P_{C}(t) \leq P_{L}(t)-P_{S}(t)$

$P_{D}(t) \leq P_{S}(t)$

where: $P_{C}(t)$ is the scheduled charging, $P_{D}(t)$ is the scheduled discharging, $r_{1}(t)$ is the output of the discharge ranking function, $r_{2}(t)$ is the output of the charge ranking function, $P_{S}(t)$ is the total scheduled power demand, $P_{L}(t)$ is the network limit for timestep $t . P N$ is the nominal power of the EV charger,V2B is the agreed depth of discharge level,BC is the EV battery capacity, $C_{e f f}$ is the charger efficiency, $S o C_{i n}$ is the initial and $S o C_{\max }$ is the maximum $\mathrm{SoC}$ level.

Unlike EVs, the ESUs are not mobile therefore they are always connected to their charging equipment. The calculation of their charging / discharging power set points is repeated every timestep (for the remaining timesteps of the day), after calculating the set points of the connected EVs. In this way the demand / generation profile of the ESUs is adjusted to the arrival/departure times of the EVs. To extend the battery life, it was assumed that the LEMS operates the ESU within a "safety" zone between $20 \%$ and $80 \%$ of the nominal storage capacity.

Triad Cost Reduction Schedule: The Triad Cost Reduction Schedule attempts to reduce the electricity cost of the building manager during triad peaks. This is achieved by shifting demand from the expensive triad timesteps to the cheap off-peak timesteps. The LEMS adjusts the charging/discharging schedules of the EVs and ESUs to an electricity price curve, and places the charging and discharging events during cheap and expensive timesteps respectively. This operation is triggered from the warnings of the Triad Prediction Tool.

Demand Response Schedule: The Demand Response Schedule aims at enabling the building manager to participate in the ancillary 


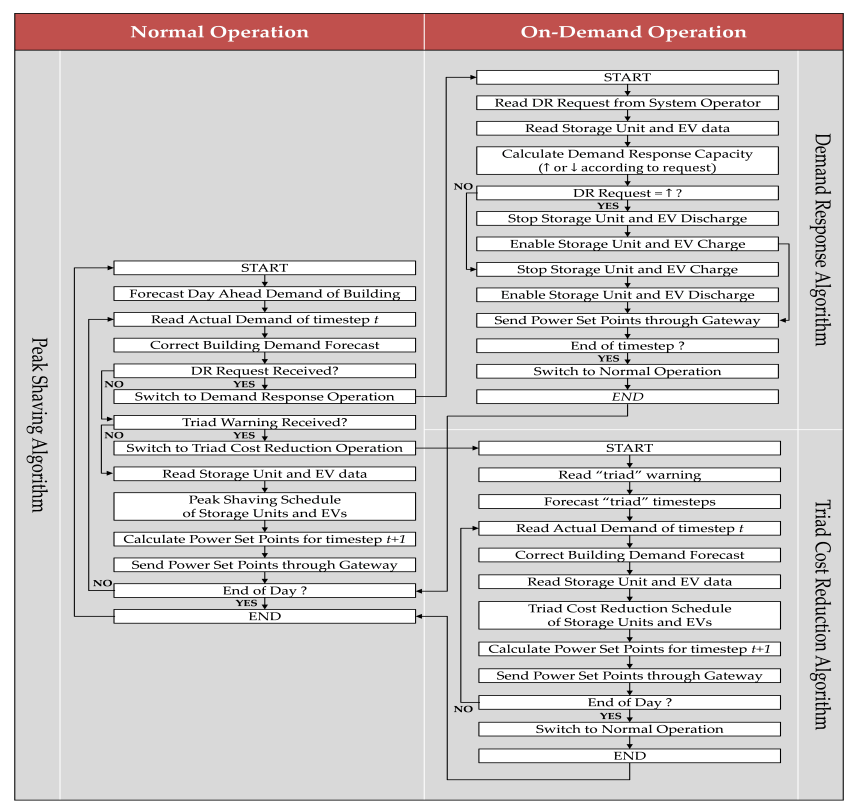

Figure 4: The operation of the LEMS

services market and provide demand response actions to the grid. It was assumed that the System Operator sends requests to the building manager to either reduce or increase its aggregated demand in the next timestep. Triggered by the arrival of such a request, the LEMS overrides the charging / discharging schedules of the available controllable assets and either starts charging all available assets or discharging them.

Operational Algorithm: The LEMS operates in two cycles, namely the Normal and the On-Demand cycle. The stages in the algorithm are illustrated in Fig. 4.

During Normal operation, the LEMS forecasts next days electricity demand of a building using the Electricity Demand Forecast Tool. The forecasted demand is updated on every timestep using the actual demand of the last timestep. In case there are no demand response requests from the System Operator or triad warnings from the Triad Prediction Tool, the LEMS uses the Peak Shaving Schedule strategy to manage the charging/discharging of the EVs and ESUs. The calculated power set points of the next timestep are forwarded to the chargers of the EVs and the ESUs through a Gateway. The cycle is repeated in every timestep, considering the new arrivals of EVs.

In case a demand response request is received, the On-Demand operation is activated and the LEMS executes the Demand Response algorithm. Depending on the availability of the EVs and ESUs, the LEMS calculates the demand response capacity at that timestep (the maximum increase/reduction of demand it can offer). In case the demand response request is to reduce the demand, the LEMS overrides the existing charging schedules and discharges the ESUs and the connected EVs and vice versa if its a demand up request. Constraints for nominal charger rates, battery capacity and SoC safety zones are applied and considered. The calculated power set points are sent to the EVs and ESUs through the Gateway. It is important to note that the Demand Response operation lasts only one timestep (the one with a demand response request), and on the next timestep the LEMS switches back to Normal operation.

In case a triad warning is received for a certain day, the LEMS switches to the On-Demand operation and executes the Triad Cost Reduction algorithm. As soon as this operation is activated, the
LEMS cancels the existing charging / discharging schedules and forecasts the timesteps where the triad peaks are most probable to occur using the Triad Prediction Tool. These timesteps are the ones where the demand should be reduced to decrease the triad charges for the building manager. The ESUs and every connected EV, as well as every other EV that arrives during that day, are scheduled to discharge at those timesteps. The charging events are scheduled according to the daily electricity prices of the building manager, always avoiding the triad timesteps.

\section{DEPLOYMENT OF LEMS}

The LEMS system has been implemented as a stand-alone environment that can be scaled based on the number of buildings being considered. A cloud-based environment has been used to realise the system, using the CometCloud system. CometCloud [21] is a cloud infrastructure specifically focused on supporting integration of distributed computer platforms. It achieves this through the use of the Comet coordination "spaces" - an abstraction based on the availability of a distributed shared memory that all users and providers can access and observe, enabling information sharing by publishing requests / offers to/for information to this shared memory (the "spaces" concept is based on a distributed Linda Tuple space). A user interested in executing a particular type of demand schedule (as discussed in section 3), can deploy a "task" in CometSpace. This task is subsequently allocated to any available resource that has the capacity to execute the task. Resources can be managed within a local cluster or outsourced to public clouds, such as Amazon.com. CometCloud deployment consists of a Master and number of Workers. The Master is responsible for managing the interaction between workers and tasks that have been submitted to the system by one or more users. A worker can directly execute a task, or act as a gateway to a third party system. Execution spaces can be created in the context of a single site to provision local resources or to support a cloudburst (i.e. when additional capacity is needed to respond to a sudden peak in demand) to public clouds or external high performance computing systems. Moreover, they can be used to create a private sub-federation across several sites. This case can be useful when several sites have some common interest and they decide to jointly target certain types of tasks as a specialized community. CometCloud is open source and has been validated in a number of scientific and financial scenarios. CometCloud has been demonstrated to work alongside specialist computing environments (such as large scale computing clusters that are part of the US TeraGrid and XSEDE projects) and public Cloud systems from Amazon.com.

We consider the following cloud deployments to support LEMS: (a) Data from EV/ESU is received by the CometCloud Master, and depending on the type of schedule requested, a Worker manages the execution required. In this deployment, the user (in this case the building manager) knows which type of schedule they are interested in beforehand.

(b)Data from EV/ESU is received by the CometCloud Master, and a multiple Worker nodes are deployed to execute each of the three possible schedules. The user then decides which schedule is most appealing. This approach enables multiple concurrently scenarios to be established before the user makes a decision.

(c) Data form EV/ESU is received by the CometCloud Master, and multiple Worker nodes are used to calculate a number of potential what-if scenarios - such as: (i) likelihood of a triad peak occurring on a particular day; (ii) likelihood of a number of EVs arriving / departing from the building facility; (iii) likelihood of a particular weather profile existing on a given day. Such what-if scenarios can 


\begin{tabular}{|c|c|c|c|}
\hline & TRIAD DATE & \multicolumn{2}{|c|}{ Triad Half-Hour } \\
\hline \multirow{7}{*}{$\sqrt{1}$} & Arrival times & Mean: 08:00 & StdDev: $0.75 \mathrm{~h}$ \\
\hline & Departure times & Mean: 19:00 & StdDev: $1 \mathrm{~h}$ \\
\hline & Battery Capacity & Mean: $27 \mathrm{kWh}$ & StdDev: 3kWh \\
\hline & $\begin{array}{l}\text { SoC upon } \\
\text { connection }\end{array}$ & Mean: $84 \%$ & StdDev: $4 \%$ \\
\hline & V2B & \multicolumn{2}{|c|}{$15 \%$} \\
\hline & Nominal power & \multicolumn{2}{|c|}{$3 \mathrm{~kW}$} \\
\hline & Charger Efficiency & \multicolumn{2}{|c|}{0.95} \\
\hline \multirow{6}{*}{ 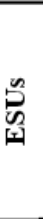 } & Initial SoC & $75 \%$ & $25 \%$ \\
\hline & Battery Capacity & $10 \mathrm{kWh}$ & $20 \mathrm{kWh}$ \\
\hline & Max SoC & \multicolumn{2}{|c|}{$80 \%$} \\
\hline & Min SoC & \multicolumn{2}{|c|}{$20 \%$} \\
\hline & Nominal power & \multicolumn{2}{|c|}{$7 \mathrm{~kW}$} \\
\hline & Charger Efficiency & \multicolumn{2}{|c|}{0.95} \\
\hline
\end{tabular}

Figure 5: EV and ESU Assumptions

be executed as concurrent tasks by multiple Worker nodes. The user then decides which scenario they would like to consider in practice. CometCloud therefore enables dynamic scaling of computational resources to execute multiple concurrent scenarios. The scalability of this system across a local cluster or via cloud bridging to a remote public cloud, enables more efficient use of the underlying computational environment.

\subsection{Description of scenarios}

Three scenarios were studied to test the performance of the proposed system, namely Peak Shaving Operation, Triad Cost Reduction Operation and Demand Response Operation. In the Peak Shaving Operation scenario the LEMS operates in Normal mode, aiming to reduce the peak demand of the building facility. In the Triad Cost Reduction scenario aim is to reduce the electricity cost occurring due to Triads for the building manager. Finally, the Demand Response scenario studies the performance of the LEMS in a demand response occasion, where two demand response requests are received from the System Operator.

A fleet of $10 \mathrm{EVs}$ was considered in all three scenarios, assuming normal distributions for their arrival/departure times, initial SoC and battery capacity. Table 5 presents the mean and standard deviation values of these distributions. It was also assumed that the EV drivers were willing to discharge up to $15 \%$ of their vehicle's battery. The building facility was assumed to have 2 ESUs with characteristics presented in Table 5.

Scenario 1: Peak Shaving Operation: Figure 6 presents the real aggregated demand of the commercial building facility when the LEMS operates under Normal operation (Peak Shaving). The EVs and the ESUs are scheduled to charge during the off-peak hours, and discharged during the peak hours. A $6.9 \%$ peak reduction was achieved comparing to the initial demand of the facility (without EVs and ESUs).

Scenario 2: Triad Cost Reduction Operation: In this scenario it is assumed that the LEMS receives a triad warning from the Triad Prediction Tool and the Triad Cost Reduction Operation is activated. The "triad" peak hours (as predicted from the Triad Prediction Tool) were found to be between 17:30 and 19:30. This information was used by the LEMS in order to schedule the charging/discharging of the EVs and ESUs and reduce the overall electricity cost for the building manager. The resulting aggregated demand of the building facility is presented in Figure 7. Due to the simultaneous charging of the EVs and ESUs during the low tariff period, the peak demand increasedWith more EVs, this peak is expected to increase and possibly stress the existing equipment.

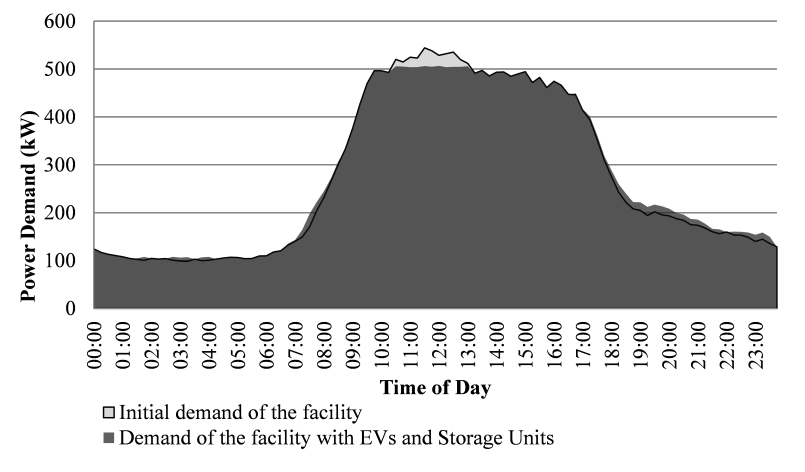

Figure 6: Demand of the facility with Peak Shaving Operation.

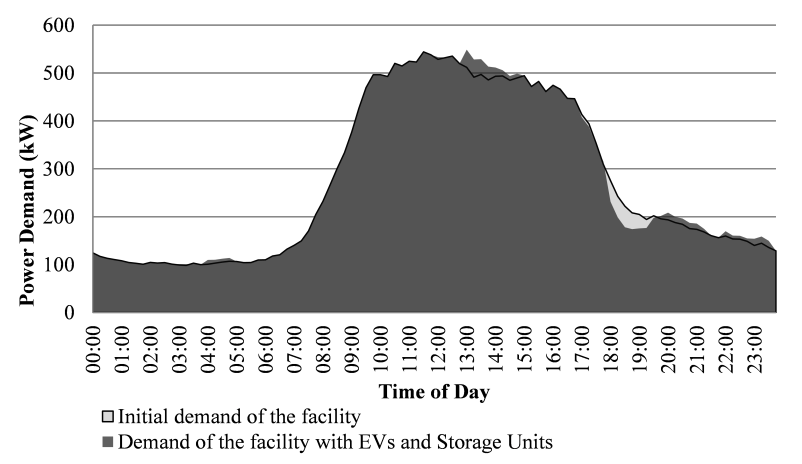

Figure 7: Demand of the facility with Triad Cost Reduction Operation.

Additional measures should be considered, like the demand cap mentioned in [29]. The discharging of the EVs/ ESUs resulted in a $7.5 \%$ reduction of the electricity requirements during the triad peak hours (17:00 -20:00). This number is expected to rise when more EVs are available, increasing the cost savings of the building manager. In this scenario discharging events are concentrated 17:30 and 19:30. After the discharging period, the LEMS tried to charge the EVs in order for them to leave the facility fully charged. However, because the discharging period was very close to their departure times, there was not enough time for them to fully recharge -6 out of $10 \mathrm{EVs}$ left the facility with a battery SoC less than $100 \%$. As this is a commercial building facility the average departure times of EVs coincides with the discharging period - a risk all EV drivers are aware of, and highlights the utility of a maximum discharge capacity agreement (V2B level) being the worst case scenario for the EV driver. This is not the case for the ESUs, as their charging / discharging schedules are not subject to time limitations due to their continuous connection to the charging stations.

Scenario 3: Demand Response Operation: In this scenario it was assumed that the LEMS receives two requests for demand response. A request was received at 08:00, asking the LEMS to reduce the demand of the building facility. A second request was received at 14:00, asking the LEMS to increase the demand of the facility.The LEMS activates this operation only for one timestep to satisfy the demand response request, and returns to the Normal Operation (Peak Shaving) at the end of it. The LEMS successfully reduced the aggregated demand of the facility at 08:00 by discharging the connected EVs and ESUs. A $17.7 \%$ demand reduction was achieved compared to the case without any EVs/ ESUs. After this, it returned to Normal Operation until the second demand response request was received at 14:00. Upon receipt of the "demand increase" request, the LEMS overrode the charging/discharging schedu- 
les of the EVs/ ESUs and charged every available unit increasing the demand by $8.9 \%$.

\section{CONCLUSION}

A Local Energy Management System is described that has been realised by a cloud-based infrastructure. The model estimates the probability of having a triad peak and forecasts the demand of the building during the time of the day that such a peak is likely to occur. Three scheduling algorithms were developed, namely Peak Shaving, Triad Cost Reduction and Demand Response. The LEMS was implemented as a software package deployed on cloud, and its operation was demonstrated in three simulation scenarios on a set of six real commercial buildings located in a science park in Manchester, UK.

In the Peak Shaving Operation the LEMS was successfull in achieving 6.9\% peak reduction. In the Triad Cost Reduction Operation the LEMS successfully resulted in a $7.5 \%$ reduction of the electricity requirements during triad peak hours (17:00 - 20:00), reducing the triad costs for the building manager. In the Demand Response Operation, the LEMS was able to reduce the overall demand by $17.7 \%$ as a response to the demand reduction request. In addition, an overall $8.9 \%$ demand increase was achieved as a response to the demand increase request.

Acknowledgement: This work was carried out in the InnovateUK/EPSRCfunded (EP/M507131/1) "Ebbs and Flows of Energy Systems" (EFES) project.

\section{REFERENCES}

[1] I. E. Agency. Renewable energy. https://www.iea.org/about/faqs/energyefficiency/, 2017.

[2] L. Bu, D. Zhao, Y. Liu, and Q. Guan. A hierarchical classification algorithm for evaluating energy consumption behaviors. In Neural Networks (IJCNN), 2014 International Joint Conference on, pages 1461-1466. IEEE, 2014.

[3] S. Chen, H. B. Gooi, and M. Wang. Sizing of energy storage for microgrids. IEEE Transactions on Smart Grid, 3(1):142-151, 2012.

[4] M. Fardadi, A. S. Ghafari, and S. Hannani. Pid neural network control of sut building energy management system. In Advanced Intelligent Mechatronics. Proceedings, 2005 IEEE/ASME International Conference on, pages 682-686. IEEE, 2005.

[5] P. Ferreira, A. Ruano, S. Silva, and E. Conceicao. Neural networks based predictive control for thermal comfort and energy savings in public buildings. Energy and Buildings, 55:238-251, 2012.

[6] N. Grid. Uk I national grid. http://www2.nationalgrid.com/uk/, 2017.

[7] X. Guan, Z. Xu, and Q.-S. Jia. Energy-efficient buildings facilitated by microgrid. IEEE Transactions on smart grid, 1(3):243-252, 2010.

[8] M. W. Khan, M. A. Choudhry, and M. Zeeshan. An efficient design of genetic algorithm based adaptive fuzzy logic controller for multivariable control of hvac systems. In Computer Science and Electronic Engineering Conference (CEEC), 2013 5th, pages 1-6. IEEE, 2013.

[9] Y. P. Kumar and R. Bhimasingu. Review and retrofitted architectures to form reliable smart microgrid networks for urban buildings. IET Networks, 4(6):338-349, 2015.

[10] J. Laustsen. Energy efficiency requirements in building codes, energy efficiency policies for new buildings. International Energy Agency (IEA), pages 477-488, 2008.
[11] J. Li, Z. Wu, S. Zhou, H. Fu, and X.-P. Zhang. Aggregator service for pv and battery energy storage systems of residential building. CSEE Journal of Power and Energy Systems, 1(4):3-11, 2015.

[12] X. Li, C. P. Bowers, and T. Schnier. Classification of energy consumption in buildings with outlier detection. IEEE Transactions on Industrial Electronics, 57(11):3639-3644, 2010.

[13] N. Liu, Q. Chen, J. Liu, X. Lu, P. Li, J. Lei, and J. Zhang. A heuristic operation strategy for commercial building microgrids containing evs and pv system. IEEE Transactions on Industrial Electronics, 62(4):2560-2570, 2015.

[14] W. Mai, C. Chung, T. Wu, and H. Huang. Electric load forecasting for large office building based on radial basis function neural network. In PES General Meeting | Conference \& Exposition, 2014 IEEE, pages 1-5. IEEE, 2014.

[15] C. Mullen, P. C. Taylor, V. Thornley, and N. S. Wade. Use of standby generation for reduction of transmission network charges for half-hourly metered customers. In Power Engineering Conference (UPEC), 2014 49th International Universities, pages 1-6. IEEE, 2014.

[16] D. T. Nguyen and L. B. Le. Optimal bidding strategy for microgrids considering renewable energy and building thermal dynamics. IEEE Transactions on Smart Grid, 5(4):1608-1620, 2014.

[17] U. of Waikato. Weka 3 - data mining with open source machine learning software in java. http://www.cs.waikato.ac.nz/ml/weka/, 2017.

[18] L. Pérez-Lombard, J. Ortiz, and C. Pout. A review on buildings energy consumption information. Energy and buildings, 40(3):394-398, 2008.

[19] M. Pipattanasomporn, M. Kuzlu, and S. Rahman. An algorithm for intelligent home energy management and demand response analysis. IEEE Transactions on Smart Grid, 3(4):2166-2173, 2012.

[20] U. N. E. Programme. .:: United nations environment programme (unep) - sbci ::. http://www.unep.org/sbci/AboutSBCI/Background.asp, 2017.

[21] Rutgers. Cometcloud. http://nsfcac.rutgers.edu/CometCloud/node/7, 2017.

[22] J. Van Roy, N. Leemput, F. Geth, J. Büscher, R. Salenbien, and J. Driesen. Electric vehicle charging in an office building microgrid with distributed energy resources. IEEE Transactions on sustainable energy, 5(4):1389-1396, 2014.

[23] L. Wang, Z. Wang, and R. Yang. Intelligent multiagent control system for energy and comfort management in smart and sustainable buildings. IEEE transactions on smart grid, 3(2):605-617, 2012.

[24] Z. Wang and L. Wang. Adaptive negotiation agent for facilitating bi-directional energy trading between smart building and utility grid. IEEE Transactions on Smart Grid, 4(2):702-710, 2013.

[25] T. Weng and Y. Agarwal. From buildings to smart buildings -sensing and actuation to improve energy efficiency. IEEE Design \& Test of Computers, 29(4):36-44, 2012.

[26] P. Zhao, S. Suryanarayanan, and M. G. Simoes. An energy management system for building structures using a multi-agent decision-making control methodology. IEEE Transactions on Industry Applications, 49(1):322-330, 2013. 